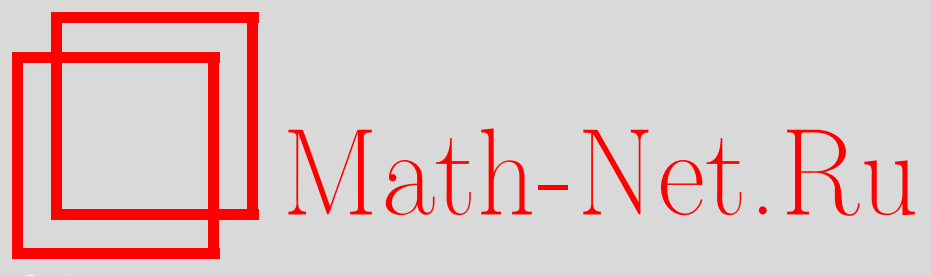

О. Е. Галкин, О последовательностях операторов композиции в пространствах функций ограниченной $\Phi$ вариации, Матем. заметки, 2009, том 85, выпуск 3, 330341

DOI: https://doi.org/10.4213/mzm3891

Использование Общероссийского математического портала Math-Net.Ru подразумевает, что вы прочитали и согласны с пользовательским соглашением http://www.mathnet.ru/rus/agreement

Параметры загрузки:

IP : 54.166 .219 .16

26 апреля 2023 г., 13:30:55

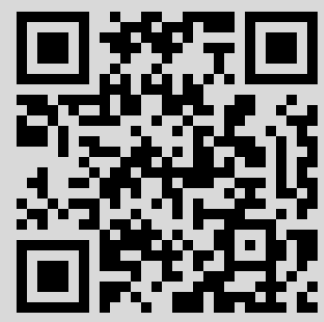


Том 85 выпуск 3 март 2009

УДК $517.518 .24+517.518 .3$

\section{О последовательностях операторов композиции \\ в пространствах функций ограниченной Ф-вариации}

\section{О. Е. Галкин}

Основные результаты работы содержатся в теоремах 1 и 2 . В теореме 1 найдены необходимые и достаточные условия на последовательность функций $h_{n}:\langle c, d\rangle \rightarrow\langle a, b\rangle, n=1,2, \ldots$, при которых для любой функции $f:\langle a, b\rangle \rightarrow \mathbb{R}$, имеющей конечную $\Phi$-вариацию, ограничена последовательность $\Psi$-вариаций $\left\{V_{\Psi}\left(\langle c, d\rangle ; f \circ h_{n}\right)\right\}_{n=1}^{\infty}$, вычисленных для композиций функций $f$ и $h_{n}$. В теореме 2 то же сделано для последовательности функций $h_{n}: \mathbb{R} \rightarrow \mathbb{R}, n=1,2, \ldots$, и последовательности $\Psi$-вариаций $\left\{V_{\Psi}\left(\langle a, b\rangle ; h_{n} \circ f\right)\right\}_{n=1}^{\infty}$.

Библиография: 10 названий.

\section{1. Предварительные сведения}

\section{1. Основные определения и обозначения.}

ОПРЕДЕЛЕНИЕ 1 [1], [2]. 1) Назовем $\varphi$-функиией непрерывную неограниченную неубывающую функцию $\Phi:[0,+\infty) \rightarrow[0,+\infty)$ такую, что $\Phi(x)=0$ только при $x=0$.

$2)$ Будем говорить, что $\varphi$-функция $\Phi$ удовлетворяет $\Delta_{2}$-условию около нуля, если существуют такие $\delta>0$ и $K>0$, что $\Phi(2 x) \leqslant K \Phi(x)$ при всех $x \in[0, \delta]$ (что, очевидно, равносильно существованию такого $K_{1}>0$, что $\Phi(2 x) \leqslant K_{1} \Phi(x)$ при всех $x \in[0,1])$.

ОПРЕДЕЛЕНИЕ 2. Промежутком $\Pi=\langle c, d\rangle$ при $c \leqslant d$ будем называть каждое из подмножеств $[c, d],(c, d),[c, d),(c, d]$ вещественной оси $\mathbb{R}$. Пустое и одноточечные множества также будем считать промежутками.

Всюду далее $\Phi$ и $\Psi-\varphi$-функции, $\langle a, b\rangle$ и $\langle c, d\rangle$ - непустые промежутки.

Обозначим символом $\tau\langle a, b\rangle$ множество всех разбиений $T=\left\{t_{0}, t_{1}, \ldots, t_{n}\right\}$ промежутка $\langle a, b\rangle$ (здесь $n \in \mathbb{N} \cup\{0\}, a \leqslant t_{0}<t_{1}<\cdots<t_{n} \leqslant b$ ).

ОПРЕДЕЛЕНИЕ 3 (см. п. 1.2). Ф-вариащией функции $f:\langle a, b\rangle \rightarrow \mathbb{R}$ по промежутку $\langle a, b\rangle$ называется величина

$$
V_{\Phi}(\langle a, b\rangle ; f)=\sup \left\{V_{\Phi}[T ; f] \mid T \in \tau\langle a, b\rangle\right\},
$$

где $V_{\Phi}[T ; f]=\sum_{i=1}^{n} \Phi\left(\left|f\left(t_{i}\right)-f\left(t_{i-1}\right)\right|\right)-$ предвариация функции $f$ по разбиению $T=\left\{t_{0}, t_{1}, \ldots, t_{n}\right\}$ промежутка $\langle a, b\rangle$.

Работа выполнена при поддержке Российского фонда фундаментальных исследований (гранты №№ 03-01-00473, 06-01-00761).

(C) О.Е. ГАлкин, 2009 
Символом $B V_{\Phi}\langle a, b\rangle$ обозначим множество всех функций $f:\langle a, b\rangle \rightarrow \mathbb{R}$, имеющих конечную $\Phi$-вариацию на $\langle a, b\rangle$.

Если $\Phi(x) \equiv x$, то будем записывать $V_{\Phi}(\langle c, d\rangle ; g)$ как $V(\langle c, d\rangle ; g)$ и $B V_{\Phi}\langle c, d\rangle$ как $B V\langle c, d\rangle$.

ОПРЕДЕЛЕНИЕ 4. Модулем непреръвности функции $h: \mathbb{R} \rightarrow \mathbb{R}$ на отрезке $[u, v]$ называется функция $\omega_{h}([u, v]):[0, \infty) \rightarrow[0, \infty]$, определяемая равенством

$$
\omega_{h}([u, v] ; \delta)=\sup \{|h(t)-h(s)||t, s \in[u, v],| t-s \mid \leqslant \delta\} .
$$

Для каждого $N \in \mathbb{N}$ обозначим через $\mathbf{J}_{N}\langle c, d\rangle$ семейство всех подмножеств промежутка $\langle c, d\rangle$, которые можно представить в виде объединения $N$ промежутков (ср. [3]). Тогда $\mathbf{J}\langle c, d\rangle=\bigcup_{N=1}^{\infty} \mathbf{J}_{N}\langle c, d\rangle$ - набор подмножеств из $\langle c, d\rangle$, представимых в виде объединения конечного числа промежутков.

Если $E \in \mathbf{J}\langle c, d\rangle$, то обозначим через $|E|$ наименьшее число промежутков, объединение которых дает $E$.

Множество всех функций $g:\langle c, d\rangle \rightarrow \mathbb{R}$ таких, что прообраз $g^{-1}(\Pi)$ принадлежит $\mathbf{J}_{N}\langle c, d\rangle$ для любого промежутка $\Pi \subset \mathbb{R}$, обозначим через $\mathbf{F} \mathbf{J}_{N}\langle c, d\rangle$. Ясно, что $\mathbf{F J}_{N}\langle c, d\rangle \subset \mathbf{F J}_{N+1}\langle c, d\rangle$. Символом $\mathbf{F} \mathbf{J}\langle c, d\rangle$ обозначим объединение $\bigcup_{N=1}^{\infty} \mathbf{F} \mathbf{J}_{N}\langle c, d\rangle$.

ОПРЕДЕЛЕНИЕ 5. Будем говорить, что множества $A$ и $B$ из класса $\mathbf{J}\langle c, d\rangle$ omдeлень (друг от друга), если между любыми двумя промежутками, один из которых входит в $A$, а другой - в $B$, найдется хотя бы одна точка, не принадлежащая ни одному из этих промежутков.

ПримеР. Множества $(0 ; 1)$ и $(1 ; 2)$ отделены друг от друга, а множества $(0 ; 1]$ и $(1 ; 2)$ - нет.

ОПРЕДЕЛЕНИЕ 6. Назовем функцию $g:\langle c, d\rangle \rightarrow\langle a, b\rangle$ кусочно-постоянной, если $\langle c, d\rangle$ можно разбить на конечное число непересекающихся промежутков, на каждом из которых $g$ постоянна (это равносильно тому, что $g$ принадлежит $\mathbf{F} \mathbf{J}\langle c, d\rangle$ и принимает конечное число значений).

В настоящей работе мы исследуем, при каких условиях на последовательность функций $h_{n}:\langle c, d\rangle \rightarrow\langle a, b\rangle$ (соответственно $\left.h_{n}: \mathbb{R} \rightarrow \mathbb{R}\right), n=1,2, \ldots$, последовательность операторов правой композиции $f \mapsto f \circ h_{n}$ (соответственно операторов левой композиции $\left.f \mapsto h_{n} \circ f\right)$ на пространстве $B V_{\Phi}\langle a, b\rangle$ поточечно ограничена по $\Psi$-вариации, т.е. для любой функции $f$ из класса $B V_{\Phi}\langle a, b\rangle$ ограничена последовательность $\Psi$-вариаций $\left\{V_{\Psi}\left(\langle c, d\rangle ; f \circ h_{n}\right)\right\}_{n=1}^{\infty}\left(\right.$ соответственно $\left.\left\{V_{\Psi}\left(\langle a, b\rangle ; h_{n} \circ f\right)\right\}_{n=1}^{\infty}\right)$.

1.2. Об истории проблемы. Напомним, что впервые понятие вариации функции было введено Жорданом [4], а понятие $\Phi$-вариации функции было определено: Винером [5] для $\Phi(x)=x^{2}$, Марцинкевичем [6] и Янгом [7] для $\Phi(x)=x^{p}, p \geqslant 1$, и Янгом [8] в общем случае. Пространства функций ограниченной вариации с различных точек зрения изучались, в частности, Мущелаком и Орличем [1], Лещневичем и Орличем [2], Чистяковым и Галкиным [9].

Джозефи [3] показал, что если $h:[0 ; 1] \rightarrow[0 ; 1]$, то

1) $h \circ f \in B V[0 ; 1]$ для любой функции $f \in B V[0 ; 1]$ тогда и только тогда, когда $h$ - липшицева;

2) $f \circ h \in B V[0 ; 1]$ для любой функции $f \in B V[0 ; 1]$ тогда и только тогда, когда $h \in \mathbf{F J}[0 ; 1]$. 
Цемночоловский и Орлич [10; теорема 1] доказали, что если функция $\Psi$ удовлетворяет $\Delta_{2}$-условию около нуля и $h_{n}: \mathbb{R} \rightarrow \mathbb{R}, h_{n}(0)=0$ для $n=1,2, \ldots$, то условие

a) $\sup _{n \in \mathbb{N}} V_{\Psi}\left(\langle a, b\rangle ; h_{n} \circ f\right)<\infty$ для любой $f \in B V_{\Phi}\langle a, b\rangle$ равносильно условию

б) для любого $v>0$ существует вещественное $K_{v}>0$ такое, что при всех $n=$ $1,2, \ldots$ выполняется неравенство

$$
\Psi\left(\left|h_{n}\left(u_{2}\right)-h_{n}\left(u_{1}\right)\right|\right) \leqslant K_{v} \Phi\left(\left|u_{2}-u_{1}\right|\right), \quad u_{1}, u_{2} \in[-v, v] .
$$

Этот результат является обобщением результата 1) Джозефи.

\section{2. Основные результаты}

\section{1. Функции $h_{n}$ действуют справа.}

Tеорема 1. Пусть $h_{n}:\langle c, d\rangle \rightarrow\langle a, b\rangle$ для $n=1,2, \ldots$. Тогда условие

а) $\sup _{n \in \mathbb{N}} V_{\Psi}\left(\langle c, d\rangle ; f \circ h_{n}\right)<\infty$ для любой $f \in B V_{\Phi}\langle a, b\rangle$ равносильно условию

б) существует такое натуральное $N$, что $h_{n} \in \mathbf{F J}_{N}\langle c, d\rangle$ при всех $n=1,2, \ldots$, и если $\sup _{0<x<1} \Psi(x) / \Phi(x)=\infty$, то при каждом $n=1,2, \ldots$ бункиия $h_{n}$ принимает не более $N$ значений.

Кроме того, если выполнено условие б), то

1) если $\sup _{0<x<1} \Psi(x) / \Phi(x)<\infty$, то для любых $f \in B V_{\Phi}\langle a, b\rangle$ и всех $n=1,2, \ldots$ выполняется оченка

$$
V_{\Psi}\left(\langle c, d\rangle ; f \circ h_{n}\right) \leqslant 2 K_{f} N \cdot V_{\Phi}(\langle a, b\rangle ; f),
$$

где $K_{f}=\sup \left\{\Psi(z) / \Phi(z) \mid z>0, \Phi(z) \leqslant V_{\Phi}(\langle a, b\rangle ; f)\right\}<\infty ;$

2) если $\sup _{0<x<1} \Psi(x) / \Phi(x)=\infty$, mо $f \circ h_{n} \in B V_{\Psi}\langle c, d\rangle$ для любой $\varphi$-функции $\Psi$, причем

$$
V_{\Psi}\left(\langle c, d\rangle ; f \circ h_{n}\right) \leqslant N^{2} \Psi\left(M_{f}\right),
$$

где $M_{f}=\sup \{|f(x)-f(y)| \mid x, y \in\langle c, d\rangle\}<\infty$.

СлеДСТвИЕ. Пусть $h:\langle c, d\rangle \rightarrow\langle a, b\rangle$. Тогда условие

а) $f \circ h \in B V_{\Psi}\langle c, d\rangle$ для любой $f \in B V_{\Phi}\langle a, b\rangle$

равносильно условию

б) $h \in \mathbf{F} \mathbf{J}\langle c, d\rangle$, и если $\sup _{0<x<1} \Psi(x) / \Phi(x)=\infty$, то $h$ принимает лишь конечное число значений (т.е. кусочно-постоянна).

Эти утверждения обобщают результат 2) Джозефи.

\section{2. Функции $h_{n}$ действуют слева.}

Tеорема 2. Eсли $h_{n}: \mathbb{R} \rightarrow \mathbb{R}$ при $n=1,2, \ldots$, то условие

a) $\sup _{n \in \mathbb{N}} V_{\Psi}\left(\langle a, b\rangle ; h_{n} \circ f\right)<\infty$ для любой $f \in B V_{\Phi}\langle a, b\rangle$

равносильно условию

б) для любого $v>0$ существует вещественное $K_{v}>0$ mакое, что при всех $n=1,2, \ldots$ выполняется неравенство

$$
\Psi\left(\left|h_{n}\left(u_{2}\right)-h_{n}\left(u_{1}\right)\right|\right) \leqslant K_{v} \Phi\left(\left|u_{2}-u_{1}\right|\right), \quad u_{1}, u_{2} \in[-v, v] .
$$


Если в классе $B V_{\Phi}\langle a, b\rangle$ есть хотя бы одна непрерьвная функция, не являющаяся постоянной, то условия а) и б) равносильны (формально более слабому) условию

$\left.\mathrm{a}^{\prime}\right) \sup _{n \in \mathbb{N}} V_{\Psi}\left(\langle a, b\rangle ; h_{n} \circ f\right)<\infty$ для любой непрерьвной $f \in B V_{\Phi}\langle a, b\rangle$.

Эта теорема лишь немного усиливает результат Цемночоловского и Орлича (элиминировано $\Delta_{2}$-условие около нуля на функцию $\left.\Psi\right)$.

ЗАмЕчАниЕ. 1) В [10; с. 434] показано, что если выполнено условие б), то для любых $f \in B V_{\Phi}\langle a, b\rangle$ и всех $n=1,2, \ldots$ выполняется оценка

$$
V_{\Psi}\left(\langle a, b\rangle ; f \circ h_{n}\right) \leqslant K_{v} \cdot V_{\Phi}(\langle a, b\rangle ; f) .
$$

2 ) Если функция $\Psi$ строго возрастает, то неравенство (1), очевидно, равносильно неравенству

$$
\omega_{h_{n}}([-v, v] ; \delta) \leqslant \Psi^{-1}\left(K_{v} \Phi(\delta)\right), \quad \delta \in[-v, v] .
$$

3) Если $\Psi(L x) / \Psi(x) \rightarrow \infty$ при $L \rightarrow+\infty$ равномерно по $x$ из некоторого интервала $(0, \varepsilon)$ (это верно, например, для $\Psi(x)=|x|^{p}, p>0$, и любого $\varepsilon>1$ ), то из (2) следует, что при некотором $L>0$ выполнено неравенство

$$
\omega_{h_{n}}([-v, v] ; \delta) \leqslant L \cdot \Psi^{-1}(\Phi(\delta)), \quad \delta \in[-v, v] .
$$

4) Если $\Phi(x)=\Psi(x)=1 / \ln (1 / x)$ при $0<x<\delta_{0}$ для некоторого $0<\delta_{0}<1$, то неравенство (2) влечет гёльдеровость функции $h_{n}$, поскольку $\Psi^{-1}\left(K_{v} \Phi(\delta)\right)=\delta^{1 / K_{v}}$ при $0<\delta<\delta_{0}$.

5) Согласно лемме 10 (см. далее раздел 3$)$ в классе $B V_{\Phi}\langle a, b\rangle$ тогда и только тогда найдется хотя бы одна непрерывная функция, не являющаяся постоянной, когда для некоторого $C>0$ при всех $x \in[0,1]$ выполнено неравенство $\Phi(x) \leqslant C x$.

\section{3. Вспомогательные утверждения}

Перед изложением вспомогательных утверждений приведем таблицу зависимостей между ними:

\begin{tabular}{|c|l||c|l|}
\hline № леммы & Где применяется & № леммы & Где применяется \\
\hline 1 & леммы 2 и 9, теорема 1 & 6 & лемма 8 \\
\hline 2 & лемма 7 & 7 & теорема 1 \\
\hline 3 & лемма 7 & 8 & теорема 1 \\
\hline 4 & леммы 7 и 8 & 9 & теорема 1 \\
\hline 5 & лемма 7, теорема 2 & 10 & теорема 2 \\
\hline
\end{tabular}

Лемма 1. Пусть $g \in \mathbf{F J}_{N}\langle c, d\rangle u\left|g\left(x_{1}\right)-g\left(x_{2}\right)\right| \leqslant M$ npu $x_{1}, x_{2} \in\langle c, d\rangle$ (например, $g(x) \in[0, M]$ при $x \in\langle c, d\rangle)$. Тогда

а) $V(\langle c, d\rangle ; g) \leqslant M(2 N-1)$, причем оченка точна для любого $N \in \mathbb{N}$;

б) если функиия $g$ принимает не более $N$ значений, $\operatorname{mo~} g \in B V_{\Psi}\langle c, d\rangle$ для любой ө-функиии $\Psi$, причем

$$
V_{\Psi}(\langle c, d\rangle ; g) \leqslant\left(N^{2}-1\right) \cdot \Psi(M) .
$$

Доказательство (ср. [3; лемма 1]). а) От противного. Пусть $V(\langle c, d\rangle ; g)>$ $M(2 N-1)$. Тогда существует разбиение $T$ такое, что $V[T ; g]=\sum_{i=1}^{n}\left|g\left(t_{i}\right)-g\left(t_{i-1}\right)\right|>$ $M(2 N-1)$. Так как $\left|g\left(t_{i}\right)-g\left(t_{i-1}\right)\right| \leqslant M$, найдется, по крайней мере, $2 N$ различных 
интервалов с концами $g\left(t_{i_{j}}\right)$ и $g\left(t_{i_{j}-1}\right), j=1, \ldots, 2 N$, имеющих непустое пересечение. Пусть $z$ - точка из этого пересечения. Тогда прообраз хотя бы одного из отрезков $[z, g(c)+M],[z, g(c)-M]$ состоит из не менее, чем $N+1$ промежутков. Это противоречит условию леммы.

Теперь докажем точность оценки. Положим $g(x)=M N \cdot x$ при $x \in[0 ; 1 / N)$, и продолжим функцию $g$ периодически с периодом $1 / N$ на полуинтервал $[0 ; 1)$. Тогда $g \in \mathbf{F J}_{N}[0 ; 1)$ и $V([0 ; 1) ; g)=M(2 N-1)$.

б) Так как функция $g$ постоянна на промежутках, количество которых не более $N^{2}$, то в сумме $\sum_{i=1}^{n} \Psi\left(\left|g\left(t_{i}\right)-g\left(t_{i-1}\right)\right|\right)=V_{\Psi}[T ; g]$ имеется не более $N^{2}-1$ ненулевых слагаемых. Каждое из них не превосходит $\Psi(M)$. Поэтому

$$
V_{\Psi}(\langle c, d\rangle ; g)=\sup \left\{V_{\Psi}[T ; g] \mid T \in \tau\langle c, d\rangle\right\} \leqslant\left(N^{2}-1\right) \Psi(M) .
$$

Лемма доказана.

Лемма 2. Характеристическая функиия $\chi_{E}$ множества $E \subset\langle c, d\rangle$ имеет конечную $\Phi$-вариацию по $\langle c, d\rangle$ тогда и только тогда, когда $E$ есть обгединение конечного числа промежутков, т.е. $E \in \mathbf{J}\langle c, d\rangle$. При этом для любого $K \in \mathbb{R}$ выполнено неравенство

$$
V_{\Phi}\left(\langle c, d\rangle ; K \cdot \chi_{E}\right) \geqslant 2 \Phi(|K|) \cdot(|E|-1) .
$$

ДокАзАтЕльство. 1) Если $E$ есть объединение конечного числа промежутков, то функция $\chi_{E}$ кусочно-постоянна, поэтому согласно пункту б) леммы 1 имеем $V_{\Phi}\left(\langle c, d\rangle ; \chi_{E}\right)<\infty$.

2) Обратно, пусть $V_{\Phi}\left(\langle c, d\rangle ; \chi_{E}\right)<\infty$. Так как для любого разбиения $T$ величина $V_{\Phi}\left[T ; \chi_{E}\right]=\sum_{i=1}^{n} \Phi\left(\left|\chi_{E}\left(t_{i}\right)-\chi_{E}\left(t_{i-1}\right)\right|\right)$ принимает лишь значения вида $k \cdot \Phi(1)$, где $k \in\{0,1,2, \ldots\}$, то $V_{\Phi} \chi_{E}=V_{\Phi}\left[T_{0} ; \chi_{E}\right]$ для некоторого разбиения $T_{0}=\left\{t_{0}<t_{1}<\right.$ $\left.\cdots<t_{n}\right\}$. Тогда

a) $\chi_{E}(t)=\chi_{E}\left(t_{0}\right)$ для любого $t<t_{0}, t \in\langle c, d\rangle$, так как иначе $V_{\Phi}\left[\{t\} \cup T_{0} ; \chi_{E}\right]>$ $V_{\Phi}\left[T_{0} ; \chi_{E}\right]$

б) аналогично, $\chi_{E}\left(t_{n}\right)=\chi_{E}(t)$ для любого $t>t_{n}, t \in\langle c, d\rangle$;

в) если $\chi_{E}\left(t_{i-1}\right)=\chi_{E}\left(t_{i}\right)$, то $\chi_{E}(t)=\chi_{E}\left(t_{i}\right)$ при любом $t \in\left(t_{i-1}, t_{i}\right)$, так как иначе $V_{\Phi}\left[T_{0} \cup\{t\} ; \chi_{E}\right]>V_{\Phi}\left[T_{0} ; \chi_{E}\right]$;

г) если $\chi_{E}\left(t_{i-1}\right) \neq \chi_{E}\left(t_{i}\right)$, то положим $s_{i}=\inf \left\{s \in\left(t_{i-1}, t_{i}\right] \mid \chi_{E}(s)=\chi_{E}\left(t_{i}\right)\right\}$. Тогда, во-первых, $\chi_{E}\left(t_{i-1}\right)=\chi_{E}(t)$ при $t \in\left(t_{i-1}, s_{i}\right)$; во-вторых, $\chi_{E}(t)=$ $\chi_{E}\left(t_{i}\right)$ при $t \in\left(s_{i}, t_{i}\right)$, так как иначе $V_{\Phi}\left[T_{0} \cup\{s, t\} ; \chi_{E}\right]>V_{\Phi}\left[T_{0} ; \chi_{E}\right]$, где $s \in$ $\left(s_{i}, t\right)$ такое, что $\chi_{E}(s)=\chi_{E}\left(t_{i}\right)$.

Итак, $E$ состоит из промежутков с концами в точках $s_{i}$ или в $\pm \infty$, так что этих промежутков не более $n$ штук.

3) Неравенство $V_{\Phi}\left(\langle c, d\rangle ; K \cdot \chi_{E}\right) \geqslant 2 \Phi(|K|) \cdot(|E|-1)$ получается из оценки $V_{\Phi}[T$; $\left.K \cdot \chi_{E}\right] \geqslant 2 \Phi(|K|) \cdot(|E|-1)$, где $T=\left\{t_{1}<s_{1}<\cdots<s_{n-1}<t_{n}\right\}$, точки $t_{1}, \ldots, t_{n}$ лежат в $E$, а точки $s_{1}, \ldots, s_{n-1}-$ вне $E$.

Лемма 3. Пусть любые два множества из данной последовательности множеств или не пересекаются, или вложены (множество с большим номером вложено в множество с менъшим номером). Тогда из этой последовательности можно выделить подпоследовательность попарно непересекающихся множеств или подпоследовательность вложенных множеств. 
ДокАЗАтельство. Пусть $\left\{S_{n}\right\}$ - данная последовательность. Множество $S_{m}$, в которое вложено лишь конечное число множеств из $\left\{S_{n}\right\}$, назовем множеством с конечным вложением. Рассмотрим два случая.

1) В $\left\{S_{n}\right\}$ имеется бесконечное число множеств с конечным вложением. Покажем, что тогда существует подпоследовательность $\left\{S_{n_{k}}\right\}$ попарно непересекающихся множеств. Действительно, в каждое множество с конечным вложением вложено хотя бы одно множество, не содержащее других множеств из $\left\{S_{n}\right\}$. Поэтому существует бесконечная подпоследовательность $\left\{S_{n_{k}}\right\}$ множеств, не содержащих других множеств из $\left\{S_{n}\right\}$. Очевидно, элементы в $\left\{S_{n_{k}}\right\}$ попарно не пересекаются.

2) В $\left\{S_{n}\right\}$ имеется лишь конечное число множеств с конечным вложением. Покажем, что тогда существует подпоследовательность $\left\{S_{n_{k}}\right\}$ вложенных множеств.

В качестве $n_{1}$ возьмем номер, начиная с которого каждое $S_{n}$ содержит бесконечное число других множеств из $\left\{S_{n}\right\}$. Далее строим $\left\{n_{k}\right\}$ по индукции: если $n_{k}$ уже найдено, то $S_{n_{k}}$ содержит некоторое $S_{n_{k+1}}$ с номером $n_{k+1}>n_{k}$.

Лемма доказана.

Лемма 4. а) Из любого набора $\left\{E_{1}, \ldots, E_{N \cdot n^{2}}\right\}$, содержащего $N \cdot n^{2}$ попарно не пересекающихся множеств из класса $\mathbf{J}_{N}\langle a, b\rangle$, можно выбрать $n$ множеств $E_{j_{1}}, \ldots, E_{j_{n}}$, которые попарно отделены друг от друга.

б) Из любой последователъности $\left\{E_{n}\right\} \subset \mathbf{J}\langle a, b\rangle$, элементы которой попарно не перекаются друг с другом, можно выбрать подпоследовательность множеств, которые попарно отделены друг от друга.

ДокАзАтЕЛЬСтво. а) Применим индукцию по $n$. Для $n=1$ утверждение очевидно.

Пусть утверждение верно для $n-1$. Тогда из множеств $E_{j}, j=1, \ldots, N \cdot(n-1)^{2}$, можно выбрать $(n-1)$ множество $E_{j_{1}}, \ldots, E_{j_{n-1}}$, которые попарно отделены друг от друга. Объединение выбранных множеств имеет не более $N(2 n-2)$ граничных точек. Значит, среди $N(2 n-1)$ оставшихся множеств $E_{j}$ мы можем найти множество $E_{j_{n}}$, которое отделено от множеств $E_{j_{1}}, \ldots, E_{j_{n-1}}$.

б) Снова используем индукцию по $n$. На первом шаге положим $n_{1}=1, E_{n_{1}}=E_{1}$.

Далее, пусть множества $E_{n_{1}}, \ldots, E_{n_{k}}$ уже выбраны. У составляющих их интервалов в совокупности лишь конечное число граничных точек, поэтому от выбранных множеств может быть не отделено лишь конечное число множеств $E_{n}$ с номерами $n>n_{k}$. Значит, существует $E_{n_{k+1}}$, отделенное от множеств $E_{n_{1}}, \ldots, E_{n_{k}}$.

Лемма 5. Пусть $\left\{a_{n}\right\}$ u $\left\{b_{n}\right\}-$ числовые последовательности такие, что $\left\{a_{n}\right\}$ положительна, $\lim _{n \rightarrow \infty} a_{n}=0 u \lim _{n \rightarrow \infty} b_{n} / a_{n}=\infty$. Тогда существуют строго возрастающие последовательности натуральных чисел $\left\{n_{k}\right\} u\left\{M_{k}\right\}$ такие, что $\sum_{k=1}^{\infty} M_{k} a_{n_{k}}<\infty$, но $M_{k} b_{n_{k}} \rightarrow \infty, k \rightarrow \infty$.

ДокАЗАтЕльство. Положим $\varepsilon_{k}=1 / 2^{k}, k=1,2, \ldots$. Тогда $\sum_{k=1}^{\infty} \varepsilon_{k}<\infty$. Возьмем $M_{1}=n_{1}=1$. Далее по индукции строим для каждого натурального $k>1$ номер $n_{k}$ так, что $a_{n_{k}}<\varepsilon_{k} / M_{k-1}, b_{n_{k}} / a_{n_{k}}>k / \varepsilon_{k}$ и $n_{k}>n_{k-1}$. Затем выбираем натуральное $M_{k}$ так, что $\varepsilon_{k} / a_{n_{k}} \leqslant M_{k}<\varepsilon_{k} / a_{n_{k}}+1$. Тогда $M_{k}>M_{k-1}, M_{k} a_{n_{k}}<2 \varepsilon_{k}$ и $M_{k} b_{n_{k}}>k$ при $k>1$.

Поэтому $\sum_{k=1}^{\infty} M_{k} a_{n_{k}}<2 \sum_{k=1}^{\infty} \varepsilon_{k}<\infty$, но $M_{k} b_{n_{k}} \rightarrow \infty, k \rightarrow \infty$.

ЛЕмма 6. Если $\sup _{0<x<1} \Psi(x) / \Phi(x)=\infty$, то существуют последовательность $\left\{x_{k}\right\}$ различных точек из интервала $(0 ; 1)$, сходящаяся $\kappa 0$, и последовательность $\left\{M_{k}\right\} \subset \mathbb{N}$ maкие, что $\sum_{k=1}^{\infty} M_{k} \Phi\left(x_{k}\right)<\infty$, но $M_{k} \Psi\left(x_{k}\right) \rightarrow \infty, k \rightarrow \infty$. 
ДокАЗАтЕЛьство. Из условия следует, что существует последовательность $\left\{t_{n}\right\}$ различных точек из $(0 ; 1)$ такая, что $\Psi\left(t_{n}\right) / \Phi\left(t_{n}\right) \rightarrow \infty, n \rightarrow \infty$. Так как $\Psi$ ограничена на $(0 ; 1)$, то $\Phi\left(t_{n}\right) \rightarrow 0, n \rightarrow \infty$. Отсюда, так как $\Phi-\varphi$-функция, следует, что $\left\{t_{n}\right\}$ сходится к 0 . Далее остается воспользоваться леммой 5 для $a_{n}=\Phi\left(t_{n}\right)$, $b_{n}=\Psi\left(t_{n}\right)$ и положить $x_{k}=t_{n_{k}}, k=1,2, \ldots$.

Лемма 7. Eсли $h_{n}:\langle c, d\rangle \rightarrow\langle a, b\rangle, n=1,2, \ldots, u \sup _{n \in \mathbb{N}} V_{\Psi}\left(\langle c, d\rangle ; f \circ h_{n}\right)<\infty \partial \Omega \Omega$ любой $f \in B V_{\Phi}\langle a, b\rangle$, то существует такое натуральное $N$, что $h_{n} \in \mathbf{F} \mathbf{J}_{N}\langle c, d\rangle$ при всех $n=1,2, \ldots$.

ДоказАтельство. а) Докажем, что для любого промежутка $\Pi \subset\langle a, b\rangle$ множества $h_{n}^{-1}(\Pi), n=1,2, \ldots$, состоят из конечного числа промежутков, причем это число ограничено равномерно по $n \in \mathbb{N}$.

Так как $\chi_{\Pi} \in B V_{\Phi}\langle a, b\rangle$ и $\chi_{\Pi} \circ h_{n}=\chi_{h_{n}^{-1}(\Pi)}$ в силу условия леммы выполнено $\sup _{n \in \mathbb{N}} V_{\Psi}\left(\langle c, d\rangle ; \chi_{h_{n}^{-1}(\Pi)}\right)<\infty$. Поэтому в силу леммы 2 множество $h_{n}^{-1}(\Pi)$ есть объединение конечного числа промежутков, $n=1,2, \ldots$, причем

$$
2 \Psi(1) \cdot\left(\sup _{n \in \mathbb{N}}\left|h_{n}^{-1}(\Pi)\right|-1\right) \leqslant \sup _{n \in \mathbb{N}} V_{\Psi}\left(\langle c, d\rangle ; f \circ h_{n}\right)<\infty .
$$

Следовательно, величина $\left|h_{n}^{-1}(\Pi)\right|$ ограничена равномерно по $n \in \mathbb{N}$.

б) По пункту а) для любого промежутка $\Pi \subset\langle a, b\rangle$ найдется такое натуральное $N=N(\Pi)$, что $h_{n}^{-1}(\Pi)$ состоит из не более чем $N(\Pi)$ промежутков, $n=1,2, \ldots$. Докажем от противного, что можно выбрать одно и то же $N$ для всех промежутков $\Pi \subset\langle a, b\rangle$. Доказательство разобьем на семь шагов $\left.\left.\sigma_{1}\right)-\sigma_{7}\right)$.

$\left.\sigma_{1}\right)$ Если это не так, то для любого $N \in \mathbb{N}$ существуют промежуток $\Pi_{N} \subset\langle a, b\rangle$ и номер $n_{N}$ такие, что $\left|h_{n_{N}}^{-1}\left(\Pi_{N}\right)\right|>2 N^{2}$.

$\left.\sigma_{2}\right)$ По индукции построим такую последовательность промежутков $\left\{J_{N}\right\}$, что для любых номеров $N$ и $m$ при $N<m$ либо $J_{m} \subset J_{N}$, либо $J_{m} \cap J_{N}=\varnothing$, причем $\left|h_{n_{N}}^{-1}\left(J_{N}\right)\right|>N$.

На первом шаге положим $J_{1}=\Pi_{1}$. Далее, пусть $J_{1}, \ldots, J_{N-1}$ уже построены. Они разбивают промежуток $\Pi_{N}$ на не более чем $2 N$ непересекающихся промежутков $\Pi_{N}^{(1)}, \ldots, \Pi_{N}^{(m)}, m \leqslant 2 N$. Так как $2 N^{2}<\left|h_{n_{N}}^{-1}\left(\Pi_{N}\right)\right| \leqslant \sum_{k=1}^{m}\left|h_{n_{N}}^{-1}\left(\Pi_{N}^{(k)}\right)\right|$, имеем $\left|h_{n_{N}}^{-1}\left(\Pi_{N}^{\left(k_{0}\right)}\right)\right|>N$ для некоторого $k_{0}$. Полагаем $J_{N}=\Pi_{N}^{\left(k_{0}\right)}$.

$\left.\sigma_{3}\right)$ По лемме 3 из $\left\{J_{N}\right\}$ можно выделить подпоследовательность промежутков $J_{N_{k}}$, которые либо попарно не пересекаются, либо вложены. В первом случае положим $I_{k}=J_{N_{k}}, i_{k}=n_{N_{k}}$ для всех $k \in \mathbb{N}$. Тогда $\left|h_{i_{k}}^{-1}\left(I_{k}\right)\right|>k$ для любого $k \in \mathbb{N}$.

$\left.\sigma_{4}\right)$ Покажем, что во втором случае тоже можно подобрать последовательности попарно непересекающихся промежутков $\left\{I_{k}\right\}$ и номеров $\left\{i_{k}\right\}$ такие, что $\left|h_{i_{k}}^{-1}\left(I_{k}\right)\right|>k$. По пункту а) для любого промежутка $J_{N_{k}}$ величина $\left|h_{n}^{-1}\left(J_{N_{k}}\right)\right|$ ограничена равномерно по $n \in \mathbb{N}$. Поэтому мы можем выбрать из $\left\{N_{k}\right\}$ подпоследовательность $\left\{m_{k}\right\}$ так, что $\left|h_{i_{k}}^{-1}\left(J_{m_{k+1}}\right)\right|>\left|h_{i_{k}}^{-1}\left(J_{m_{k}}\right)\right|+2 k$, где $\left\{i_{k}\right\}=\left\{n_{m_{k+1}}\right\}$. Для каждого $k=1,2, \ldots$ множество $J_{m_{k}} \backslash J_{m_{k+1}}$ состоит из двух непересекающихся промежутков, поэтому $J_{m_{k+1}}=J_{m_{k}} \backslash\left(J_{k}^{(1)} \cup J_{k}^{(2)}\right)$, где $J_{k}^{(1)}$ и $J_{k}^{(2)}$ - промежутки (промежуток может быть и пустым). Отсюда

$$
\left|h_{i_{k}}^{-1}\left(J_{m_{k+1}}\right)\right| \leqslant\left|h_{i_{k}}^{-1}\left(J_{m_{k}}\right)\right|+\left|h_{i_{k}}^{-1}\left(J_{k}^{(1)}\right)\right|+\left|h_{i_{k}}^{-1}\left(J_{k}^{(2)}\right)\right| .
$$

Поэтому для некоторого $j_{k} \in\{1,2\}$ выполнено неравенство $\left|h_{i_{k}}^{-1}\left(J_{k}^{\left(j_{k}\right)}\right)\right|>k$. Полагаем $I_{k}=J_{k}^{\left(j_{k}\right)}, k \in \mathbb{N}$. 
$\left.\sigma_{5}\right)$ Согласно лемме 4,б) можно перейти к подпоследовательностям и считать промежутки $I_{k}$ попарно отделенными друг от друга.

$\left.\sigma_{6}\right)$ Подберем последовательность $\left\{z_{k}\right\} \subset(0,+\infty)$ такую, что $\sum_{k=1}^{\infty} \Phi\left(z_{k}\right)<\infty$. Снова переходя, если нужно, к подпоследовательностям (в $\left\{i_{k}\right\}$ и $\left.\left\{I_{k}\right\}\right)$, добьемся выполнения неравенств $\left|h_{i_{k}}^{-1}\left(I_{k}\right)\right|>k / \Psi\left(z_{k}\right)+1$ для $k=1,2, \ldots$.

$\left.\sigma_{7}\right)$ Зададим функцию $f_{0}$ равенством $f_{0}=\sum_{k=1}^{\infty} z_{k} \chi_{I_{k}}$. Тогда, с одной стороны, $f_{0} \in B V_{\Phi}\langle a, b\rangle$, так как $V_{\Phi}\left(\langle a, b\rangle ; f_{0}\right) \leqslant 2 \sum_{k=1}^{\infty} \Phi\left(z_{k}\right)<\infty$, но с другой стороны, $\sup _{n \in \mathbb{N}} V_{\Psi}\left(\langle c, d\rangle ; f_{0} \circ h_{n}\right)=\infty$, так как по лемме 2 при $k=1,2, \ldots$ имеем

$$
V_{\Psi}\left(\langle c, d\rangle ; f_{0} \circ h_{i_{k}}\right) \geqslant V_{\Psi}\left(\langle c, d\rangle ; z_{k} \chi_{I_{k}} \circ h_{i_{k}}\right) \geqslant 2 \Psi\left(z_{k}\right) \cdot\left(\left|h_{i_{k}}^{-1}\left(I_{k}\right)\right|-1\right)>2 k .
$$

Наличие функции $f_{0}$ с такими свойствами противоречит условию леммы.

ЛЕмма 8. Пусть выполнены следующие условия:

1) $h_{n} \in \mathbf{F J}_{N}\langle c, d\rangle, n=1,2, \ldots$, для некоторого $N \in \mathbb{N}$;

2) $\sup _{n \in \mathbb{N}} V_{\Psi}\left(\langle c, d\rangle ; f \circ h_{n}\right)<\infty$ для любой функиии $f \in B V_{\Phi}\langle a, b\rangle$;

3) $\sup _{0<x<1} \Psi(x) / \Phi(x)=\infty$.

Тогда существует такое $M \in \mathbb{N}$, что при каждом $n=1,2, \ldots$ функиия $h_{n}$ принимает не более $M$ значений.

ДокАзАтЕЛьство. а) По лемме 6 можно подобрать последовательность $\left\{z_{k}\right\} \subset$ $(0 ; 1)$ так, что $\sum_{k=1}^{\infty} M_{k} \Phi\left(z_{k}\right)<\infty$, но $\sup _{k \in \mathbb{N}} M_{k} \Psi\left(z_{k}\right)=\infty$.

б) Пусть доказываемое утверждение неверно. Тогда для любого $M \in \mathbb{N}$ существует функция $h_{n(M)}$, принимающая более чем $M$ различных значений.

в) По индукции выберем для каждого $k=1,2, \ldots$ функцию $h_{n_{k}}$ и набор попарно различных точек $y_{1}^{(k)}, \ldots, y_{M_{k}}^{(k)}$ в множестве $h_{n_{k}}(\langle c, d\rangle)$ ее значений таким образом, чтобы эти наборы попарно не пересекались, и чтобы при каждом $k$ полные прообразы $h_{n_{k}}^{-1}\left(\left\{y_{1}^{(k)}\right\}\right), \ldots, h_{n_{k}}^{-1}\left(\left\{y_{M_{k}}^{(k)}\right\}\right)$ этих точек были попарно отделены друг от друга. А именно, в качестве $h_{n_{1}}$ возьмем функцию, принимающую более чем $N \cdot M_{1}^{2}$ различных значений. Тогда по пункту а) леммы 4 среди этих значений можно выбрать $M_{1}$ точек $y_{1}^{(1)}, \ldots, y_{M_{1}}^{(1)}$ так, чтобы их полные прообразы $h_{n_{1}}^{-1}\left(\left\{y_{1}^{(1)}\right\}\right), \ldots, h_{n_{1}}^{-1}\left(\left\{y_{M_{1}}^{(1)}\right\}\right)$ были попарно отделены друг от друга. Далее, если функции $h_{n_{j}}$ и точки $y_{1}^{(j)}, \ldots, y_{M_{j}}^{(j)}$, $j=1,2, \ldots, k-1$, уже выбраны, то в качестве $h_{n_{k}}$ возьмем функцию, принимающую более чем $\left(M_{1}+\cdots+M_{k-1}+N \cdot M_{k}^{2}\right)$ различных значений. Среди них найдутся $N \cdot M_{k}^{2}$ значений, отличных от уже выбранных точек $y_{i}^{(j)}, i=1,2, \ldots, M_{j}, j=1,2, \ldots, k-1$. По лемме 4, a) из этих значений можно выбрать $M_{k}$ точек $y_{1}^{(k)}, \ldots, y_{M_{k}}^{(k)}$ так, чтобы их полные прообразы $h_{n_{k}}^{-1}\left(\left\{y_{1}^{(k)}\right\}\right), \ldots, h_{n_{k}}^{-1}\left(\left\{y_{M_{k}}^{(k)}\right\}\right)$ были попарно отделены друг от друга.

г) Далее зададим функцию $f$ так: $f\left(y_{i}^{(k)}\right)=z_{k}$ при $i=1, \ldots, M_{k}, k=1,2, \ldots$, и $f(x)=0$ в других точках $x$ промежутка $\langle a, b\rangle$. Тогда

$$
V_{\Phi}(\langle a, b\rangle ; f) \leqslant 2 \sum_{k=1}^{\infty} M_{k} \Phi\left(z_{k}\right)<\infty
$$

но $V_{\Psi}\left(\langle c, d\rangle ; f \circ h_{n}\right) \geqslant \sup _{k \in \mathbb{N}} M_{k} \Psi\left(z_{k}\right)=\infty$.

Получили противоречие. Лемма доказана. 
ЛЕмма 9. Если $g \in \mathbf{F J}_{N}\langle c, d\rangle u \sup _{0<x<1} \Psi(x) / \Phi(x)<\infty$, mo $f \circ g \in B V_{\Psi}\langle c, d\rangle$ для любой функиии $f \in B V_{\Phi}\langle a, b\rangle$, причем выполняется оценка

$$
V_{\Psi}(\langle c, d\rangle ; f \circ g) \leqslant 2 K_{f} N \cdot V_{\Phi}(\langle a, b\rangle ; f),
$$

где $K_{f}=\sup \left\{\Psi(z) / \Phi(z) \mid z>0, \Phi(z) \leqslant V_{\Phi}(\langle a, b\rangle ; f)\right\}<\infty$.

ДокАЗАТЕЛЬСтво. Так как по условию $\Psi(|f(x)-f(y)|) \leqslant K_{f} \cdot \Phi(|f(x)-f(y)|)$ при $x, y \in\langle a, b\rangle$, для любого разбиения $T \in \tau\langle c, d\rangle$ имеем

$$
\left.V_{\Psi}[T ; f \circ g]=\sum_{i=1}^{n} \Psi\left(\left|f\left(g\left(t_{i}\right)\right)-f\left(g\left(t_{i-1}\right)\right)\right|\right) \leqslant K_{f} \cdot \sum_{i=1}^{n} \Phi\left(\left|f\left(g\left(t_{i}\right)\right)-f\left(g\left(t_{i-1}\right)\right)\right|\right)\right) .
$$

Поскольку $f$ лежит в классе $B V_{\Phi}\langle a, b\rangle$, ее можно представить (см. [9; теорема 3.2]) в виде $f=\lambda \circ v$, где $v(x)=V_{\Phi}(\langle a, b\rangle \cap(-\infty, x] ; f)$ - ограниченная неубывающая функция на $\mathbb{R}$, а функция $\lambda: v(\langle a, b\rangle) \rightarrow \mathbb{R}$ удовлетворяет неравенству $|\lambda(x)-\lambda(y)| \leqslant$ $\Phi^{-1}(|x-y|)$ при $x, y \in v(\langle a, b\rangle)$. Поэтому

$$
V_{\Psi}[T ; f \circ g] \leqslant K_{f} \cdot \sum_{i=1}^{n}\left|v\left(g\left(t_{i}\right)\right)-v\left(g\left(t_{i-1}\right)\right)\right| \leqslant K_{f} \cdot V(\langle c, d\rangle ; v \circ g) .
$$

Так как для любого промежутка $\Pi$ из $\mathbb{R}$ множество $v^{-1}(\Pi)$ - также промежуток и $(v \circ g)^{-1}(\Pi)=g^{-1}\left(v^{-1}(\Pi)\right)$, то функция $v \circ g$ принадлежит классу $\mathbf{F} \mathbf{J}_{N}\langle c, d\rangle$ и принимает значения из отрезка $\left[0, V_{\Phi}(\langle a, b\rangle ; f)\right]$. Значит, по пункту а) леммы 1

$$
V(\langle c, d\rangle ; v \circ g) \leqslant V_{\Phi}(\langle a, b\rangle ; f) \cdot(2 N-1),
$$

откуда

$$
V_{\Psi}(\langle c, d\rangle ; f \circ g) \leqslant 2 K_{f} N \cdot V_{\Phi}(\langle a, b\rangle ; f)
$$

Лемма доказана.

Лемма 10. Следующие свойства равносильны:

1) среди непрерывных функций, лежащих в классе $B V_{\Phi}\langle a, b\rangle$, есть хотя бы одна функция, не являющаяся постоянной;

2) существует $C>0$ такое, что $\Phi(x) \leqslant C x$ при $x \in[0,1]$;

3) $B V\langle a, b\rangle \subset B V_{\Phi}\langle a, b\rangle$.

Доказательство. а) Выведем из 1) свойство 2). Пусть $f \in B V_{\Phi}\langle a, b\rangle$ не постоянна и непрерывна на $\langle a, b\rangle$. Тогда $f(\alpha) \neq f(\beta)$ для некоторых $\alpha<\beta$ из $\langle a, b\rangle$. В силу непрерывности $f$ для любого натурального $n$ найдутся такие точки $t_{0}, t_{1}, \ldots, t_{n}$ из $[\alpha, \beta]$, что $f\left(t_{k}\right)=f(\alpha)+(f(\beta)-f(\alpha)) \cdot k / n, k=0, \ldots, n$. Пусть $T-$ разбиение, содержащее точки $t_{0}, t_{1}, \ldots, t_{n}$. Тогда

$$
V_{\Phi}[T ; f]=\sum_{k=1}^{n} \Phi\left(\left|f\left(t_{k}\right)-f\left(t_{k-1}\right)\right|\right) \geqslant \sum_{k=1}^{n} \Phi\left(\frac{1}{n}\right)=n \Phi\left(\frac{1}{n}\right) .
$$

Отсюда $\Phi(1 / n) \leqslant V_{\Phi}([a, b] ; f) / n, n=1,2, \ldots$. Далее, если $x \in(0,1]$, то $1 /(2 k)<x \leqslant$ $1 / k$ при некотором $k \in \mathbb{N}$. Поэтому

$$
\Phi(x) \leqslant \Phi\left(\frac{1}{k}\right) \leqslant \frac{V_{\Phi}([a, b] ; f)}{k} \leqslant V_{\Phi}([a, b] ; f) \cdot 2 x .
$$

б) Импликация 2) $\Rightarrow 3$ ) очевидна.

в) Из 3) следует 1), поскольку, например, непрерывная и не постоянная функция $f(t) \equiv t$ лежит в $B V\langle a, b\rangle$, а значит, и в $B V_{\Phi}\langle a, b\rangle$. 


\section{4. Доказательство основных результатов}

ДоказатеЛЬСтво теоремы 1. А) Пусть выполнено условие а). Докажем, что выполнено условие б).

Существование такого натурального $N$, что $h_{n} \in \mathbf{F J}_{N}\langle c, d\rangle$ при всех $n=1,2, \ldots$, вытекает из леммы 7.

Предположим теперь, что $\sup _{0<x<1} \Psi(x) / \Phi(x)=\infty$. Тогда по лемме 8 количество значений функции $h_{n}$ ограничено равномерно по $n \in \mathbb{N}$.

Таким образом, выполнение условия б) доказано.

Б) Теперь предположим, что выполнено условие б) теоремы, и докажем, что выполнено условие а). Для этого достаточно воспользоваться: в случае, когда $\sup _{0<x<1} \Psi(x) / \Phi(x)<\infty,-$ леммой 1 , а в случае $\sup _{0<x<1} \Psi(x) / \Phi(x)=\infty$ - леммой 9 , положив $h_{n}=h, n=1,2, \ldots$.

Теорема доказана.

ДоказательСтво теоремы 2. А) Пусть выполнено условие а). Докажем б).

Начало - пункты 1) и 2) нашего доказательства - следует доказательству теоремы 1 в [10].

1) Докажем, от противного, что на любом отрезке $[-v, v]$ величины $\left|h_{n}(x)-h_{n}(0)\right|$ ограничены равномерно по $n \in \mathbb{N}$. Если это не так, то существуют последовательности точек $\left\{u_{i}\right\} \subset[-v, v]$ и номеров $\left\{n_{i}\right\}$, а также число $u_{0} \in[-v, v]$ такие, что

$$
\left|h_{n_{i}}\left(u_{i}\right)-h_{n_{i}}(0)\right| \rightarrow \infty, \quad i \rightarrow \infty,
$$

и

$$
\Phi\left(\left|u_{i}-u_{0}\right|\right) \leqslant \frac{1}{2^{i}}, \quad i=1,2, \ldots .
$$

Мы можем считать, что $u_{0} \leqslant u_{i} \leqslant u_{1}, i=2,3, \ldots$. Пусть $a<t_{0}<t_{1}<\cdots<t_{n}<$ $\cdots<b$. Определим функцию $f_{0}$, положив $f_{0}\left(t_{0}\right)=0, f_{0}\left(t_{i}\right)=u_{i}, i=1,2, \ldots$, и $f_{0}(t)=u_{0}$ в остальных точках $t$ промежутка $\langle a, b\rangle$. Тогда

$$
V_{\Phi}\left(\langle a, b\rangle ; f_{0}\right) \leqslant \Phi\left(u_{0}\right)+\Phi\left(u_{1}\right)+2 \sum_{i=1}^{\infty} \Phi\left(\left|u_{i}-u_{0}\right|\right)<\infty .
$$

Но при этом $\sup _{n} V_{\Psi}\left(\langle a, b\rangle ; h_{n} \circ f_{0}\right)=\infty$, так как

$$
\begin{aligned}
V_{\Psi}\left(\langle a, b\rangle ; h_{n_{i}} \circ f_{0}\right) & \geqslant \Psi\left(\left|h_{n_{i}}\left(f_{0}\left(t_{i}\right)\right)-h_{n_{i}}\left(f_{0}\left(t_{0}\right)\right)\right|\right) \\
& =\Psi\left(\left|h_{n_{i}}\left(u_{i}\right)-h_{n_{i}}(0)\right|\right) \rightarrow \infty, \quad i \rightarrow \infty .
\end{aligned}
$$

Получили противоречие.

2) Продолжим доказательство условия а) снова от противного. Если а) не выполнено, то существуют последовательность индексов $\left\{n_{i}\right\}$ и последовательность интервалов $\left(u_{i}, v_{i}\right)$ из $[-v, v], i=1,2, \ldots$, такие, что

$$
K_{i}=\frac{\Psi\left(\left|h_{n_{i}}\left(v_{i}\right)-h_{n_{i}}\left(u_{i}\right)\right|\right)}{\Phi\left(v_{i}-u_{i}\right)} \rightarrow \infty, \quad i \rightarrow \infty .
$$

Отсюда, так как $\left|h_{n}(x)-h_{n}(0)\right|$ равномерно ограничены на $[-v, v]$, получаем, что $v_{i}-u_{i} \rightarrow 0, i \rightarrow \infty$.

3) Пользуясь этими выводами и леммой 5 , выберем строго возрастающие последовательности натуральных чисел $\left\{M_{i}\right\}$ и $\left\{i_{k}\right\}$ так, чтобы для $x_{k}=u_{i_{k}}, y_{k}=v_{i_{k}}$, $m_{k}=n_{i_{k}}, k=1,2, \ldots$, выполнялись следующие четыре условия $\left.\left.3 \mathrm{a}\right)-3 г\right)$ : 
3а) существует предел $\lim _{k \rightarrow \infty} x_{k}=x_{0}$ (следовательно, и $\left.\lim _{k \rightarrow \infty} y_{k}=x_{0}\right)$;

3б) при $k=1,2, \ldots$ выполняются неравенства

$$
\Phi\left(2\left|x_{k}-x_{0}\right|\right) \leqslant \frac{1}{2^{k}}, \quad \Phi\left(2\left|y_{k}-x_{0}\right|\right) \leqslant \frac{1}{2^{k}}
$$

3в) $\sum_{k=1}^{\infty} M_{k} \Phi\left(y_{k}-x_{k}\right)<\infty$, но $M_{k} \Psi\left(\left|h_{m_{k}}\left(x_{k}\right)-h_{m_{k}}\left(y_{k}\right)\right|\right) \rightarrow \infty, k \rightarrow \infty$;

зг) при $k=1,2, \ldots$ выполняются неравенства

$$
\left|x_{k}-x_{0}\right| \leqslant \frac{1}{2}, \quad\left|y_{k}-x_{0}\right| \leqslant \frac{1}{2} .
$$

4) Построим функцию $f_{0}$ на $[a, b]$ следующим образом. Возьмем последовательность $\left\{a_{n}\right\}$ из $[a, b]$ такую, что $a=a_{1}<\cdots<a_{k}<a_{k+1}<\cdots<b, \lim _{k \rightarrow \infty} a_{k}=b$. Далее, при каждом $k=1,2, \ldots$ на интервале $\left(a_{k}, a_{k+1}\right)$ выберем точки $t_{k}^{(1)}<t_{k}^{(2)}<$ $\cdots<t_{k}^{\left(M_{k}\right)}$ и положим: $f_{0}\left(t_{k}^{(j)}\right)=x_{k}, j=1, \ldots, M_{k}$, и $f_{0}(t)=y_{k}$ в остальных точках полуинтервала $\left[a_{k}, a_{k+1}\right)$. Кроме того, зададим $f_{0}(b)=x_{0}$.

$5)$ Покажем, что $f_{0} \in B V_{\Phi}\langle a, b\rangle$. Для любого разбиения $T \in \tau\langle a, b\rangle$ вклад в сумму $V_{\Phi}[T ; f]=\sum_{i=1}^{n} \Phi\left(\left|f\left(t_{i}\right)-f\left(t_{i-1}\right)\right|\right)$ тех слагаемых, в которых точки $t_{i}$ и $t_{i-1}$ принадлежат одному интервалу $\left(a_{k}, a_{k+1}\right), k=1,2, \ldots$, можно оценить через $2 \sum_{k=1}^{\infty} M_{k} \times$ $\Phi\left(y_{k}-x_{k}\right)<\infty$, а вклад тех слагаемых, в которых $t_{i}$ и $t_{i-1}$ принадлежат разным таким интервалам, можно оценить через $2 \sum_{k=1}^{\infty}\left(\Phi\left(2\left|x_{k}-x_{0}\right|\right)+\Phi\left(2\left|y_{k}-x_{0}\right|\right)\right)$. Поэтому

$$
V_{\Phi}\left(\langle a, b\rangle ; f_{0}\right) \leqslant 2 \sum_{k=1}^{\infty} M_{k} \Phi\left(y_{k}-x_{k}\right)+2 \sum_{k=1}^{\infty}\left(\Phi\left(2\left|x_{k}-x_{0}\right|\right)+\Phi\left(2\left|y_{k}-x_{0}\right|\right)\right)<\infty .
$$

6) Покажем, что $\sup _{n \in \mathbb{N}} V_{\Psi}\left(\langle a, b\rangle ; h_{n} \circ f_{0}\right)=\infty$. Действительно, в силу результатов пункта 2) имеем

$$
\begin{aligned}
V_{\Psi}\left(\langle a, b\rangle ; h_{m_{k}} \circ f_{0}\right) & \geqslant V_{\Psi}\left(\left(a_{n}, a_{n+1}\right) ; h_{m_{k}} \circ f_{0}\right) \\
& \geqslant M_{k} \Psi\left(\left|h_{m_{k}}\left(y_{k}\right)-h_{m_{k}}\left(x_{k}\right)\right|\right) \rightarrow \infty, \quad k \rightarrow \infty .
\end{aligned}
$$

Получили противоречие. Таким образом, условие б) выполнено.

$\left.\mathrm{A}^{\prime}\right)$ Пусть в классе $B V_{\Phi}\langle a, b\rangle$ есть хотя бы одна непрерывная, не постоянная функция, но б) не выполнено. Тогда можно подобрать непрерывную функцию $f_{1} \in B V_{\Phi}\langle a, b\rangle$ такую, что $\sup _{n \in \mathbb{N}} V_{\Psi}\left(\langle a, b\rangle ; h_{n} \circ f_{1}\right)=\infty$. Для этого изменим конструкцию функции $f_{0}$ из пункта 4$)$ следующим образом. При каждом $k=1,2, \ldots$ на интервале $\left(a_{k}, a_{k+1}\right)$ выберем точки $s_{k}^{(j)}, j=0,1, \ldots, M_{k}$, так, что $s_{k}^{(0)}<t_{k}^{(1)}<$ $s_{k}^{(1)}<\cdots<s_{k}^{\left(M_{k}\right)}<t_{k}^{\left(M_{k}\right)}$, и положим: $f_{1}\left(s_{k}^{(i)}\right)=y_{k}, i=0,1, \ldots, M_{k}$, и $f_{1}\left(t_{k}^{(j)}\right)=x_{k}$, $j=1, \ldots, M_{k}$. Кроме того, зададим $f_{1}(a)=y_{1}, f_{1}(b)=x_{0}$, и продолжим функцию $f_{1}$ в оставшиеся интервалы отрезка $[a, b]$ по линейности.

Построенная так функция $f_{1}$ непрерывна на $[a, b]$. Из пункта 3г) следует, что $f_{1}(t)-f_{1}(s) \leqslant 1$ при любых $t, s \in\langle a, b\rangle$. Так как по лемме $10 \Phi(x) \leqslant C x$ при $x \in[0,1]$, применяя рассуждения, аналогичные приведенным в пункте 5$)$, получим, что $V_{\Phi}\left(\langle a, b\rangle ; f_{1}\right)<\infty$. Соотношение $\sup _{n \in \mathbb{N}} V_{\Psi}\left(\langle a, b\rangle ; h_{n} \circ f_{1}\right)=\infty$ можно доказать так же, как для функции $f_{0}$ в пункте 6$)$. 
Таким образом, если в классе $B V_{\Phi}\langle a, b\rangle$ есть хотя бы одна непрерывная, но не постоянная функция, то условие б) вытекает из а'). Следовательно, условия а) и б) равносильны условию $\left.\mathrm{a}^{\prime}\right)$.

Б) Теперь предположим, что выполнено условие б) теоремы. Тогда, как показано в $[10 ;$ с. 434] выполнено и условие а).

Теорема доказана.

Автор выражает благодарность В. В. Чистякову за постановку задачи и внимание к работе.

\section{СПИСОК ЦИТИРОВАННОЙ ЛИТЕРАТУРЫ}

[1] J. Musielak, W. Orlicz, "On generalized variations. I", Studia Math., 18 (1959), 11-41.

[2] R. Lésniewicz, W. Orlicz, "On generalized variations. II", Studia Math., 45 (1973), 71-109.

[3] M. Josephy, "Composing functions of bounded variation", Proc. Amer. Math. Soc., 83:2 (1981), 354-356.

[4] C. Jordan, "Sur la série de Fourier", C. R. Acad. Sci. Paris, 92:5 (1881), 228-230.

[5] N. Wiener, "The quadratic variation of a function and its Fourier coefficients", Massachusetts J. Math., 3 (1924), 72-94.

[6] J. Marcinkiewicz, "On a class of functions and their Fourier series", Collected Papers, Państwowe Wydawnictwo Naukowe, Warsawa, 1964, 36-41.

[7] L. C. Young, "Inequalities connected with bounded $p$-th power variation in the Wiener sense and with integrated Lipschitz conditions", Proc. London Math. Soc. (2), 43 (1937), 449-467.

[8] L. C. Young, "Sur une généralisation de la notion de variation de puissance $p$-ième bornée au sens de N. Wiener, et sur la convergence des séries de Fourier", C. R. Acad. Sci. Paris, 204:7 (1937), 470-472.

[9] V.V. Chistyakov, O.E. Galkin, "Mappings of bounded $\Phi$-variation with arbitrary function $\Phi "$, J. Dynam. Control Systems, 4:2 (1998), 217-247.

[10] J. Ciemnoczołowski, W. Orlicz, "Composing functions of bounded $\varphi$-variation", Proc. Amer. Math. Soc., 96:3 (1986), 431-436.

О. Е. Галкин

Поступило

Нижегородский государственный университет

26.06.2007

им. Н. И. Лобачевского

E-mail: galkin@mm.unn.ru 